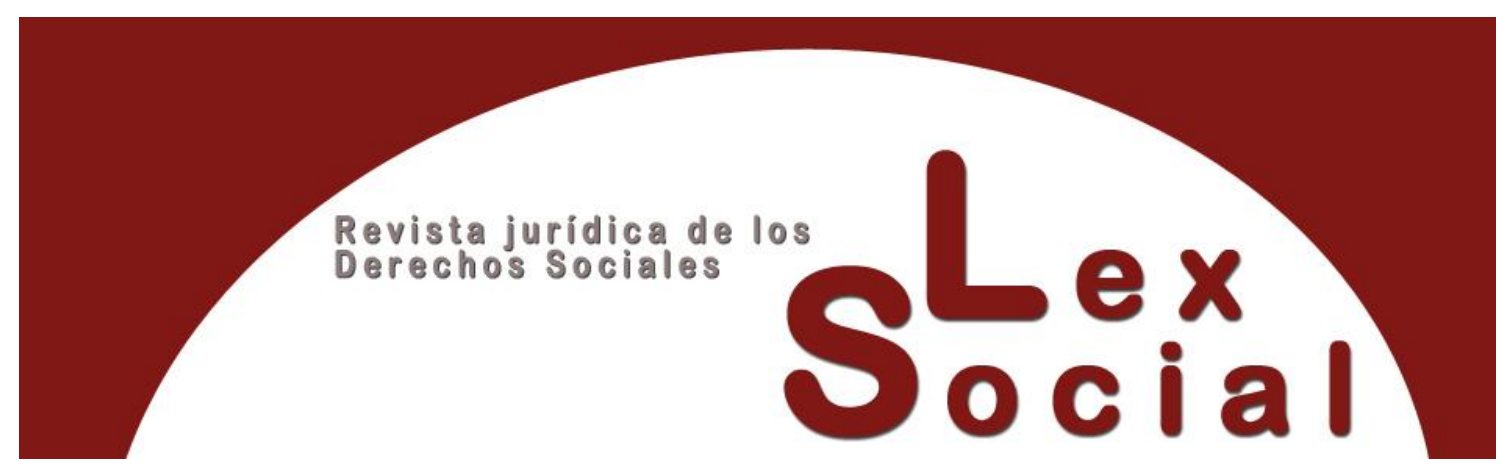

\title{
TRABALHO HUMANO NA PRIMEIRA LINHA DA PANDEMIA: ASSISTÊNCIA SÓCIO SANITÁRIA
}

\section{HUMAN WORK IN THE FIRST LINE OF THE PANDEMIC: SOCIAL AND HEALTH ASSISTANCE}

\author{
MARIA HELENA PIMENTEL \\ Unidade de Investigação em Ciências da Saúde: Enfermagem (UICISA:E) \\ Instituto Politécnico de Bragança, Portugal \\ https://orcid.org/0000-0002-0930-7469
}

Cómo citar este trabajo: Pimentel, M.H. (2021). Trabalho humano na primeira linha da pandemia: assistência sócio sanitária. Lex Social, Revista De Derechos Sociales, 11 (2), 867-878. https://doi.org/10.46661/lexsocial.5972

\section{RESUMO}

Apresenta-se em análise um problema de saúde que se prolonga há mais de um ano e não se sabe quando termina. Globalmente, a Covid-19 atingiu fortemente o setor da saúde e o setor social. Os profissionais de saúde da primeira linha têm-se revelado verdadeiros heróis na luta musculada contra o novo vírus SARS-CoV-2.

Apresentam-se como objetivos: identificar as implicações da pandemia no trabalho humano dos profissionais de saúde da primeira linha, nomeadamente a mulher como rosto deste trabalho, bem como analisar a epidemiologia da doença e a assistência socio-sanitária.

Procedemos a uma revisão da literatura sobre o tema considerando publicações desde o início da pandemia até ao momento atual.

O grave impacto da pandemia Covid-19 é explícito nos números reportados e os profissionais de saúde da primeira linha estão particularmente expostos ao risco físico e psicológico.

Os avanços científicos, a ampla divulgação para melhor conhecer o vírus e a forma de o controlar, a cooperação internacional, bem como a vacinação e os

\section{(cc) BY-NC-SA}


medicamentos mais eficazes no tratamento têm acompanhado este processo e mantido a esperança no futuro.

Palavras-chave: Covid-19, profissionais de saúde de primeira linha, riscos.

\begin{abstract}
In analysis a health problem that has lasted for more than a year and it is not known when it ends. Globally, Covid-19 has hit the healthcare and social sectors hard. First-line health professionals have proven themselves to be true heroes in this muscular fight against the new SARS-CoV-2 virus.

The objectives are: to identify the implications of the pandemic in the human work of health professionals of the first line, namely in women as the face of this work, as well as to analyze the epidemiology of the disease and socio-health care.

We proceeded to a literature review on the topic considering publications from the beginning of the pandemic to the present moment.

The serious impact of the Covid-19 pandemic is explicit in the reported numbers and first-line health professionals are particularly exposed to physical and psychological risk.

Scientific advances, widespread dissemination to better understand the virus and how to control it, international cooperation, as well as vaccination and the most effective drugs for treatment have accompanied this process and kept hope for the future.
\end{abstract}

KEYWORDS: Covid-19, first-rate health professionals, risks.

\title{
SUMARIO
}

\section{Introdução}

\subsection{Breve enquadramento do novo vírus SARS-CoV-2}

1.2. Identificar as implicações da pandemia no trabalho humano dos profissionais de saúde da primeira linha, nomeadamente na mulher como rosto deste trabalho.

1.3.Epidemiologia da doença, neste momento, e a assistência socio-sanitária

II. Reflexões finais

Bibliografia 


\section{Introdução}

\subsection{Breve enquadramento do novo vírus SARS-CoV-2}

A Covid-19 é uma doença pandémica provocada pelo novo vírus SARS-CoV-2, nunca antes identificado em seres humanos. Este agente foi identificado em dezembro de 2019, na China, mais concretamente na cidade de Wuhan e a 11 de março de 2020 foi considerada uma pandemia. A origem do vírus ainda está sob investigação e sobre a doença atualmente já se sabe um pouco mais, porém ao início sabia-se muito pouco sobre as caraterísticas deste vírus e da forma como se comportava no organismo humano. O vírus é transmitido entre pessoas, através de gotículas, secreções e aerossóis infetados. Estima-se que o período de incubação (entre a exposição e o aparecimento de sintomas) se situe entre 2 e 14 dias. A transmissão é feita por animais e pessoas, mesmo assintomáticas. A maioria dos casos apresenta sintomas respiratórios ligeiros a moderados, semelhantes à gripe sazonal. Em casos mais graves pode levar a pneumonia grave com insuficiência respiratória aguda, falência renal e de outros órgãos e eventual morte. A maioria dos infetados recupera sem sequelas ${ }^{1}$.

Esta nova doença tem provocado um enorme impacto físico e psicológico na população mundial devido ao já longo período de circulação do vírus, mais de ano de pandemia. As implicações na saúde física são bem conhecidas com o elevado número de infetados, hospitalizados e, sobretudo, o número de óbitos de grupos mais vulneráveis. Também a saúde mental é um dos aspetos a ter em conta e há quem considere a doença mental a segunda pandemia em consequência da primeira. Surtos pandémicos anteriores, como por exemplo a MERS-COVID (identificado em 2012 como agente etiológico da síndrome respiratória do médio oriente) e o Ébola, desencadearam reações psicológicas adversas nos profissionais de saúde. Este grupo profissional de primeira linha teme não só pelo próprio contágio mas também pelo medo de poder contaminar familiares e amigos e a longo prazo por implicações psicológicas provocadas por stress ansiedades e sintomas depressivos ${ }^{2,3}$.

Apresentam-se como objetivos: identificar as implicações da pandemia no trabalho humano dos profissionais de saúde da primeira linha, nomeadamente a mulher como rosto deste trabalho, bem como analisar a epidemiologia da doença e a assistência sociosanitária.

\footnotetext{
${ }^{1}$ HOFFMANN, Christian, KAMPS, Bernd Sebastian, COVID Reference, Volume 1, Hamburg, Steinhäuser Verlag, 2020

${ }^{2}$ BROOKS, Samantha et al, "The psychological impact of quarantine and how to reduce it: Rapid review of the evidence", Lancet, 2020, 395(102227), 912-20. http://doi.org/10.1016/S0140- 6736(20)30460-8

${ }^{3}$ ROGERS, Jonathan, CHESNEY, Edward and OLIVER, Dominic, "Psychiatric and neuropsychiatric presentations associated with severe coronavirus infections: a systematic review and meta-analysis with comparison to the COVID-19 pandemic", Lancet Psychiatry. 2020, 7(7), 611-627, ht tps://doi.org/10.1016/S2215- 0366(20)30203- 0
} 


\subsection{Identificar as implicações da pandemia no trabalho humano dos profissionais de saúde da primeira linha, nomeadamente na mulher como rosto deste trabalho.}

Para melhor identificar as implicações da pandemia nos profissionais de saúde e população geral assinalam-se seis características da Covid-19²:

1. Novidade - patogenia pouco conhecida. Sem tratamento eficaz, esperança na vacina para normalizar a vida de todos nós.

2. Complexidade - capaz de esgotar a capacidade de resposta dos sistemas de saúde do mundo, incluindo países com recursos elevados. Elevada taxa de mortalidade.

3. Medidas sanitárias - de isolamento e de unidade altamente diferenciadas UCIs para os casos mais graves (em Portugal, num ano aumentou para mais do dobro a capacidade destas unidades).

4. Transmissão - de fácil transmissão mesmo em assintomáticos e com longo período de incubação.

5. Impacto na saúde das populações a longo prazo, sobretudo nas doenças crónicas e na saúde mental, por adiamento de cirurgias, consultas programadas e rastreios.

6. Implicações sociais devido ao confinamento da população colocando-nos numa enorme vulnerabilidade coletiva com capacidade de pôr em causa a estrutura socioeconómica da sociedade.

Estas implicações também se repercutem nas mulheres acentuando a desigualdade de género. As mulheres foram as mais afetadas pela pandemia uma vez que são mais implicadas na perda de postos de trabalho e alvo de maior stress para articular a sua atividade profissional com o trabalho doméstico, o acompanhamento escolar dos filhos em casa e outras tarefas familiares que se intensificaram com o teletrabalho e, em muitos casos, o aumento do agregado familiar por filhos ou pais que voltaram a coabitar ${ }^{4}$. Em Portugal, foram as mulheres em $82 \%$ dos casos que ficaram em casa no apoio à família, por encerramento das escolas para controlar a Covid-19, diminuindo a sua presença no local do trabalho e desta forma comprometendo a carreira profissional, implicações que se acentuaram com a perda de rendimento uma vez que auferiram apenas $66 \%$ do salário de base. O governo de Portugal decretou majorar em $100 \%$ o apoio aos pais se ambos os progenitores assumirem partilhar a responsabilidade de apoio aos filhos de menor idade 5 .

Acresce a circunstância da maioria dos profissionais de saúde da primeira linha de atuação ter rosto feminino. A percentagem de mulheres, como resulta do Gráfico $1^{6}$, é de $83,5 \%$, clara predominância do género feminino no grupo profissional de

\footnotetext{
${ }^{4}$ GENDER DIVERSITY INDEX. European Women on Boards Gender Diversity Index, 2020.

5 REPÚBLICA PORTUGUESA, ADMINISTRAÇÃO INTERNA, Relatório sobre a Aplicação da Declaração do Estado de Emergência 16 a 30, de janeiro de 2021, Estratégia de aplicação das medidas de combate à pandemia da doença COVID-19, https://www.parlamento.pt/Documents/2021/fevereiro/Relatorio-Estado-Emergencia-16-30jan.pdf
} 
enfermeiras. De igual forma existem mais profissionais médicos do género feminino. Contudo, os médicos apresentam uma taxa de feminização $(60,6 \%)$ mais baixa (gráfico $2)^{6}$.
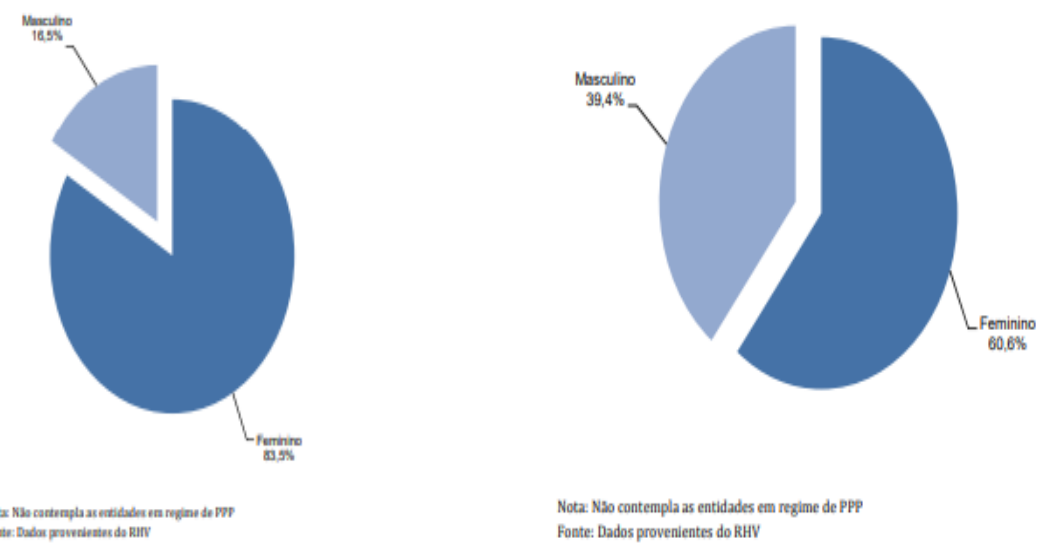

Gráfico 1. Taxa de feminização dos enfermeiros Gráfico 2. Taxa de feminização dos médicos

A necessidade de tornar visível a presença dos homens nos serviços de saúde é uma das estratégias para a efetivação de um trabalho voltado para a transformação social. Porém, a história da enfermagem em todo o Mundo está, desde as origens, associada à condição feminina. A mulher tem um sentido de cuidar. Contudo, as condições de trabalho, em regime de turnos nos hospitais e com muitas horas extra, são penosas e difíceis de encaixar nos outros papéis que as enfermeiras também assumem. Em contexto de pandemia esta circunstancia ganha maior expressão porque as condições de trabalho em segurança são a garantia de que a mulher tem a sua integridade física protegida e poderá continuar a desempenhar os seus múltiplos papéis.

A longa investigação que tem sido publicada sobre o vírus e as suas características, a forma como se comporta no organismo e a forma como progride rapidamente atingindo órgãos vitais, bem como as sequelas dos sobreviventes mais graves muito ajudaram a ajustar as respostas terapêuticas ou preventivas a este vírus. $\mathrm{O}$ facto de se ter criado uma vacina em tão curto espaço de tempo muito se deve à divulgação da sequenciação genética do vírus, à investigação e à celeridade dos $\operatorname{prazos}^{1,7.8,9,10 \text {, }}$

\footnotetext{
${ }^{6}$ REPÚBLICA PORTUGUESA, SERVIÇO NACIONAL DE SAÚDE. Relatório Social Do Ministério Da Saúde $E$ Do Serviço Nacional De Saúde 2018, https://www.sns.gov.pt/wpcontent/uploads/2019/09/Relat\%C3\%B3rio-Social-MS_SNS-2018-002.pdf
}

7 ANDERSON, RM, HEESTERBEEK, H, KLINKENBERG, D, HOLLINGSWORTH, TD, "How will country-based mitigation measures influence the course of the COVID-19 epidemic?", Lancet, 2020, 395, 931-34

${ }^{8}$ CHEN, Nanshan et al, "Epidemiological and clinical characteristics of 99 cases of 2019 novel coronavirus pneumonia in Wuhan, China: a descriptive study", Lancet, 2020, 395, 507-13.

${ }^{9}$ HUANG, Chaolin et al, "Clinical features of patients infected with 2019 novel coronavirus in Wuhan, China”, Lancet, 2020, 395, 497-506.

${ }^{10}$ KSIAZEK, TG et al, "A novel coronavirus associated with severe acute respiratory syndrome". $N$ Engl J Med. 2003, 348, 1953-1966. 
Vários estudos publicados ${ }^{1,2,3,11,12,13}$ sobre o risco da Covid-19 nos profissionais de saúde da primeira linha permitiram, igualmente, ajustar medidas de prevenção para diminuir taxas de morbilidade e mortalidade nesses profissionais.

O que se encontra também em linha de preocupação é o impacto da Covid-19 na saúde mental. Apresentamos dois estudos um realizado em Espanha outro em Portugal. O estudo espanhol ${ }^{14}$ englobou 546 trabalhadores, 296 profissionais de saúde, 105 da comunicação social, 89 comércio e 83 do serviço de proteção. Os principais resultados evidenciam que, todos os grupos, excepto os trabalhadores de serviços de proteção, mostraram níveis de impacto psicológico mais elevados do que o geral da população; trabalhadores da saúde e do comércio foram os mais afetados, 73,6\% e 65,2\%, respetivamente; as mulheres apresentaram níveis de impacto psicológico mais alto; da mesma forma nos profissionais de saúde das regiões com maiores incidências de covid19; as principais preocupações foram estar infetado pelo covid-19 ou infetar outras pessoas; o equipamento de proteção individual foi geralmente relatado como insuficiente, o que se correlacionou com níveis mais elevados de impacto psicológico; o excesso de trabalho durante a crise (sobretudo (38\%) nos profissionais da área de saúde); cuidar de pacientes com covid-19 (77\%) ou de pacientes terminais com covid19 (43,9\%). De salientar, de acordo com o estudo, maioria não recebeu apoio psicológico.

O estudo português ${ }^{15}$ visou caracterizar a saúde mental da população adulta, bem como de grupos específicos, nomeadamente profissionais de saúde e indivíduos infetados ou suspeitos de infeção pelo SARS-CoV-2 que vivenciaram episódios de quarentena, isolamento ou tratamento da Covid-19, envolveu 6079 residentes em Portugal, incluindo 2097 profissionais de saúde. A recolha de dados decorreu em dois períodos de 22 de maio a 20 de julho de 2020 e de 23 de julho a 14 de agosto. Os resultados revelam que sete em dez portugueses que estiveram em quarentena ou já recuperados da Covid19 acusaram sofrimento psicológico e mais de metade apontou sintomas de depressão moderada a grave. Das pessoas infetadas 92\% relataram sintomas de ansiedade moderada a grave e $43 \%$ sintomas de perturbação de stress pós-traumático. No caso dos profissionais de saúde, os grupos mais afetados por burnout são os que estiveram em contacto regular com doentes (33\%), os que trataram de doentes com covid-19 (43\%) e

\footnotetext{
${ }^{11}$ DEMARTINI, Karine et al. "Care for frontline health care workers in times of COVID-19", Rev. Soc. Bras. Med. Trop., 2020, vol.53, e20200358, https://doi.org/10.1590/0037-8682-0358-2020

${ }^{12}$ LAI, Jiambo et al, "Factors Associated With Mental Health Outcomes Among Health Care Workers Exposed to Coronavirus Disease 2019", JAMA, 2020, 3(3), e203976, https://doi.org/10.1001/jamanetworkopen.2020.3976.

${ }^{13}$ NGUYEN, LONG et al. "Risk of COVID-19 among front-line health-care workers and the general community: a prospective cohort study", Lancet Public Health, 2020, 5, e475-83.
}

14 RODRÍGUEZ-REY, Rocío, GARRIDO-HERNANSAIZ, Helena and BUENO-GUERRA, Nereida, "Working in the Times of COVID-19. Psychological Impact of the Pandemic in Frontline Workers in Spain”. International Journal of Environmental Research and Public Health, 2020, vol. 17, 21 8149, doi:10.3390/ijerph17218149

1515 ALMEIDA, Teresa Caldas de et al, Relatório Final: SM-Covid19 - Saúde Mental em Tempos de Pandemia, Lisboa, Instituto Nacional de Saúde Doutor Ricardo Jorge, 2020. 
os que tiveram um aumento do horário de trabalho (39\%). Este grupo 95\% aponta preocupação com a possibilidade de o país entrar numa crise económica muito grave e com a sua forma de viver não voltar a ser a mesma (76\%). Quanto às perspetivas após a pandemia, perto de metade destes profissionais sente-se otimista, sendo principalmente homens, com mais de 60 anos, com rendimentos superiores a dois mil euros e os que não tiveram necessidade de se afastar da família. De acordo com resultados deste estudo, dados da população geral, são sobretudo os jovens adultos e as mulheres que apresentam sintomas de ansiedade e de depressão moderada a grave. Já de entre os profissionais de saúde são sobretudo aqueles que trataram doentes com Covid-19 que apresentam ansiedade moderada a grave (42\%).

Atenta ao impacto da pandemia na saúde mental a $\mathrm{WHO}^{16}$ apresenta recomendações para enfrentar as consequências psicológicas e mentais do novo coronavírus por stress, risco de contaminação, incerteza, isolamento social e desemprego, entre outros motivos. Aos profissionais de saúde recomenda, entre outros aspetos, períodos de descanso, manter horários de refeições, de sono, de lazer, de exercício físico, compartilhar experiencias difíceis, comunicação com superiores e companheiros, contacto online com entes queridos afastados pelo medo do contágio e apoio psicológico.

\subsection{Epidemiologia da doença e a assistência socio-sanitária}

A Organização Internacional do Trabalho ${ }^{17}$ estabelece que "condições de trabalho seguras e saudáveis são fundamentais para um trabalho digno". Concordamos e acrescentamos que garantir a segurança e saúde no trabalho é indispensável na gestão da pandemia Covid-19. Quem nos dá conta desta problemática é a Amnistía Internacional, Public Services International and Uni Global Union ${ }^{18}$ ao contabilizar, no mundo, em março de 2021, pelo menos 17 mil mortes por Covid-19 de médicos, enfermeiros e outros trabalhadores ligados à saúde, um trabalhador a cada 30 minutos. Em alguns países onde já foram iniciados os planos de vacinação, existe o risco de os profissionais de saúde não serem considerados prioritários ou terem de esperar para ser vacinados, seja por falta de vacinas ou por problemas na implementação ou definições restritas do que se considera ser um trabalhador de saúde. Na Europa, estes profissionais foram considerados prioritários ${ }^{18}$.

Alguns paises negligenciaram a distribuição de Equipamentos de Proteção Individual (EPIs). Condições de trabalho inseguras e falta de EPIs causaram enormes problemas aos profissionais de saúde em todo o mundo, especialmente na primeira fase do aparecimento da doença ${ }^{18}$.

Em Portugal, o número de profissionais infetados desde o início da pandemia totalizam cerca de 28.000, a maioria assistentes operacionais, seguidos por enfermeiros, médicos

\footnotetext{
${ }^{16}$ World Health Organization, Novel Coronavirus - Thailand, 2020.

17 Organização Internacional do Trabalho (OIT), Trabalho digno em Portugal 2008-2018: da crise à recuperação, Genebra, BIT, 2018

${ }^{18}$ AMNISTIA INTERNACIONAL, PUBLIC SERVICES INTERNATIONAL E UNI GLOBAL UNION. Health Workers Deaths Due to COVID-19, https://www.amnistia.pt/covid-19-17-mil-profissionais-desaude-morreram-em-2021/
} 
e outros profissionais do setor. Entre os profissionais que faleceram, em número de 19, contabilizam-se 3 médicos, 1 enfermeiro, 1 técnico de diagnóstico, 2 auxiliares de saúde, 1 auxiliar de enfermagem, 2 administrativos, 1 colaborador de Estrutura Residencial para Idosos, 1 bombeiro e 7 com categoria desconhecida. Em Espanha reporta-se a 10 de dezembro de 2020, um total de 92 mortes de profissionais de saúde por Covid-19 18 .

O primeiro caso de Covid-19 confirmado em Portugal foi a 2 de março de 2020 ao contrário de Espanha que confirmou o seu primeiro caso um mês mais cedo, a 31 de janeiro de 2020. Também os gráficos 3 e 4 mostram uma epidemiologia da doença diferente entre os dois países quer em número de infetados quer em número de mortes por Covid-19. Em termos gerais, Portugal, ao contrário de Espanha, respondeu bem aos efeitos da primeira onda pandémica, porém a segunda ocorrida no outono e sobretudo a terceira em pleno inverno foram altamente marcadas pelo elevado número de infetados, número de internamento, falência do sistema nacional de saúde português, nomeadamente das unidades de cuidados intensivos e elevado número de mortes nos meses de janeiro e fevereiro de $2021^{19}$.

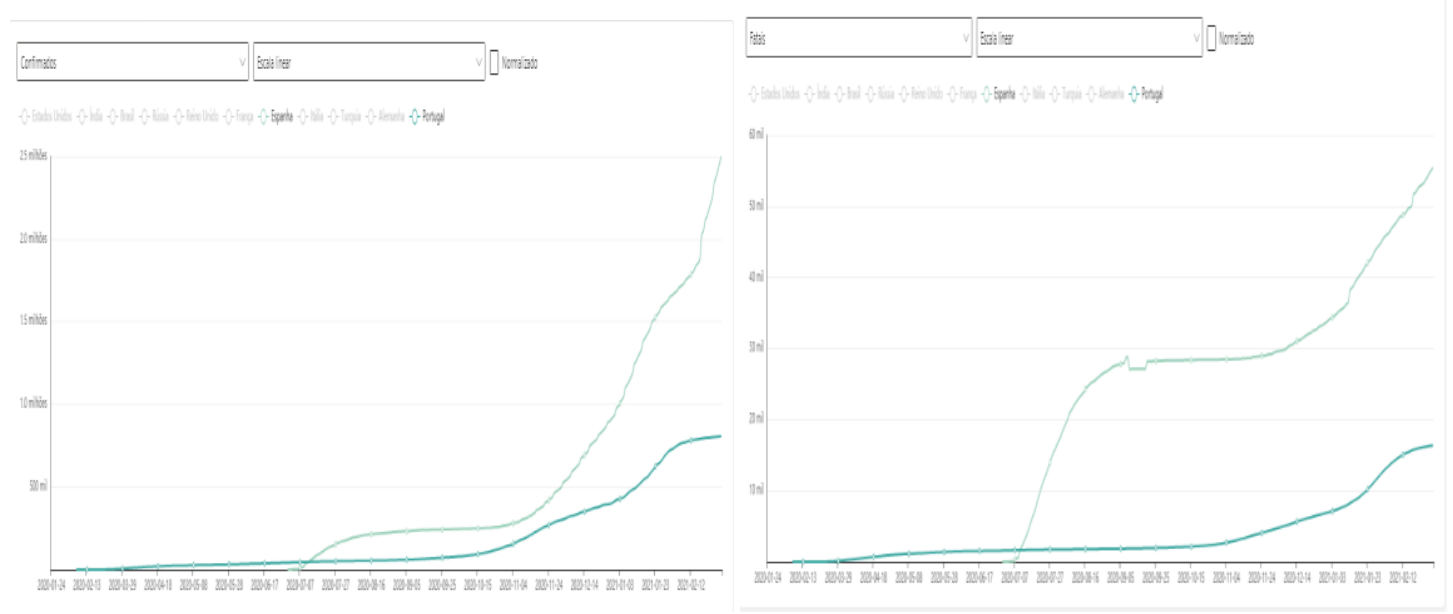

Gráfico 3. No de infetados por Covid-19 Portugal e Espanha Gráfico 4. № de mortes

Um ano após o início da pandemia fez-se o balanço. No dia 2 de março de 2021, conforme se pode observar no gráfico 5, Portugal ocupa o quarto lugar no número de infetados por Covid-19 por milhão de habitante. A lista de países com maior número de casos é encabeçada pela Republica Checa seguida da Eslováquia e Estados Unidos da América. Espanha surge em quinto e apesar de ter tido um suto mais grave na primeira onda pandémica parece ter lidado melhor com a situação ao longo do tempo ${ }^{20}$.

\footnotetext{
19 World Health Organization, Health Emergency Dashboard https://covid19.who.int/region/euro/country/pt

${ }^{20}$ SIC, Jornal da noite, 2 de março de 2021.
} 


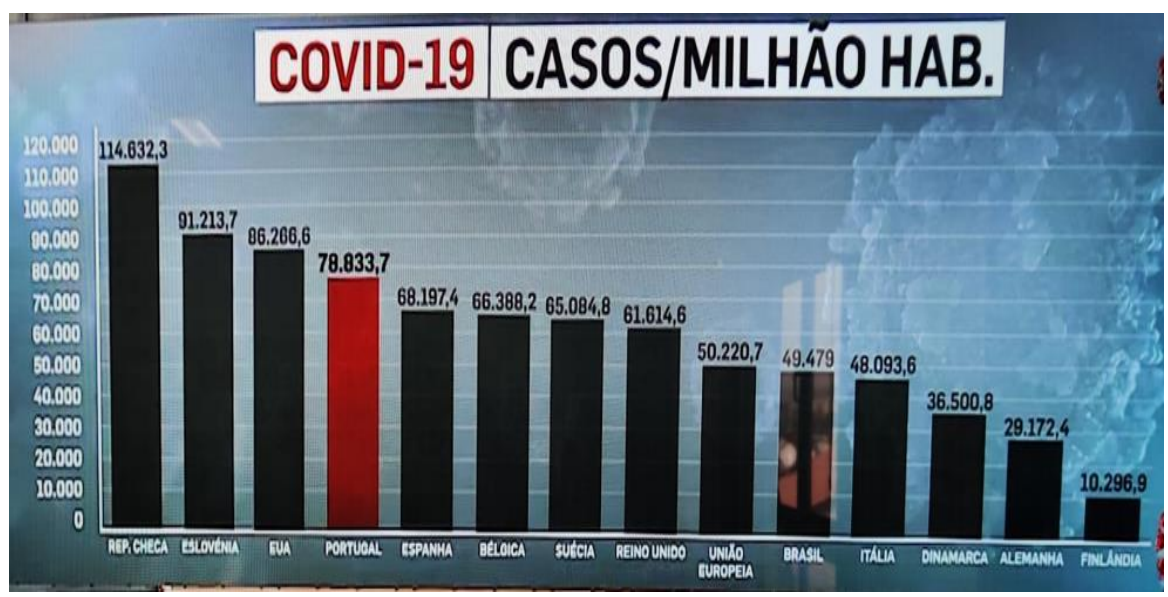

Gráfico 5. Número de casos de infetados por Covid-19/milhão de habitantes

Relativamente ao número de mortes a lista é encabeçada pela Bélgica, seguida do Reino Unido e da Itália. Portugal surge em quarto lugar acima dos Estados Unidos da América e de Espanha (gráfico 6). Esta elevada taxa de mortalidade ficou a dever-se, sobretudo, à terceira onda pandémica que esgotou os recursos sanitários do países levando à necessidade de transferir doentes de região para região e do continente para as ilhas, conforme a gestão da taxa de ocupação das unidades de cuidados intensivos. Também recorreu a ajuda externa recebendo equipas sanitárias vindas da Alemanha ${ }^{20}$.

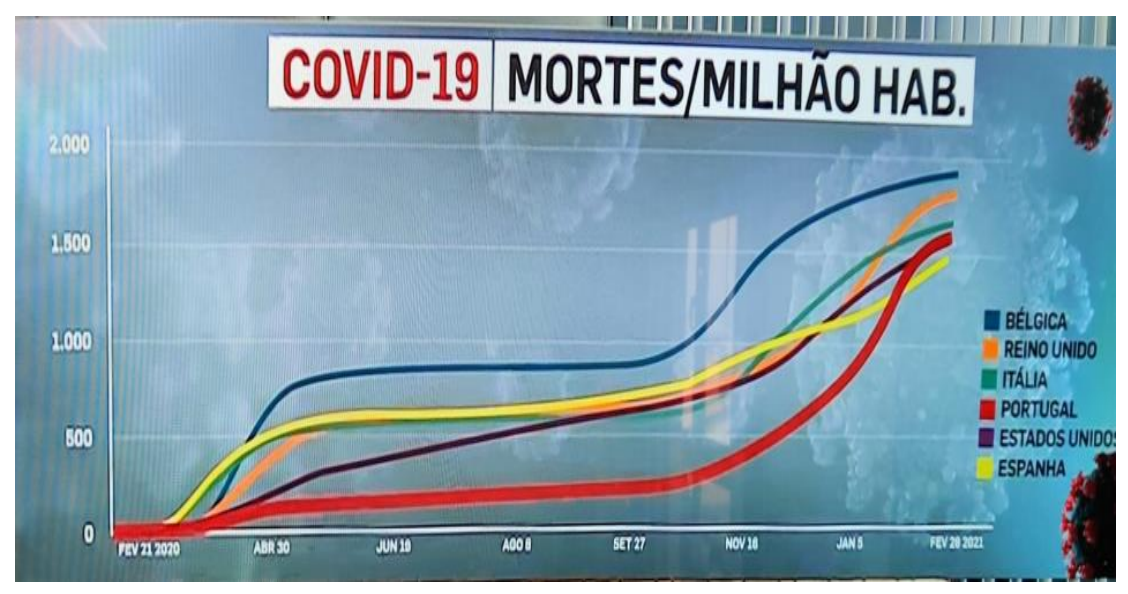

Gráfico 6. Número de mortes por Covid-19/milhão de habitantes

Em Portugal verificou-se ainda, durante o ano de pandemia em análise, um excesso de mortalidade geral, encabeçando a lista neste indicador, comparando com a média de mortalidade entre 2015 e $2019^{20}$. 


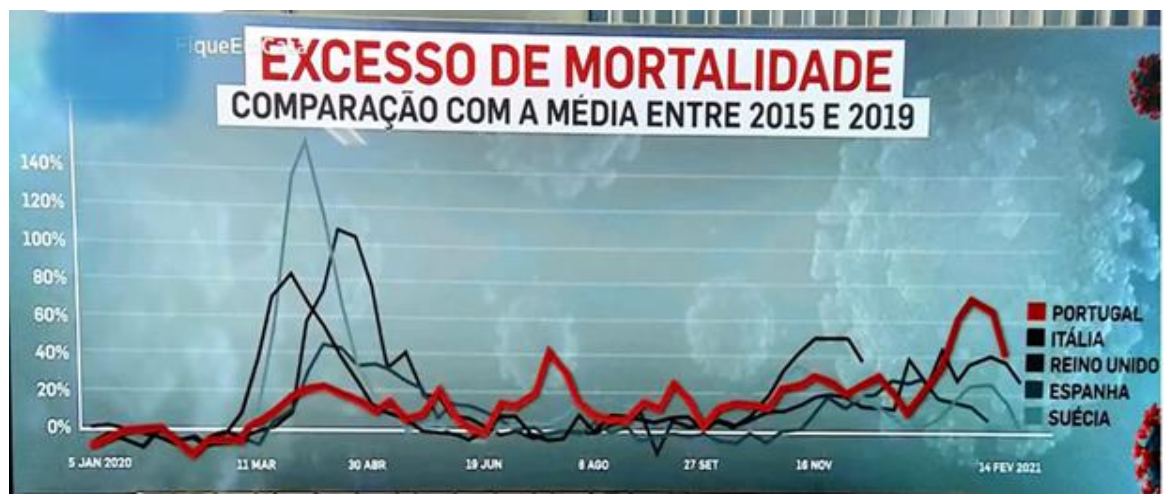

Gráfico 7. Excesso de mortalidade comparação com a média entre 2015 e 2019

Já no que diz respeito aos testes Covid-19, como se observa no gráfico 8, Portugal aumentou o número de testes nos primeiros meses deste ano de 2021, no pico da terceira onda da pandemia ${ }^{20}$.

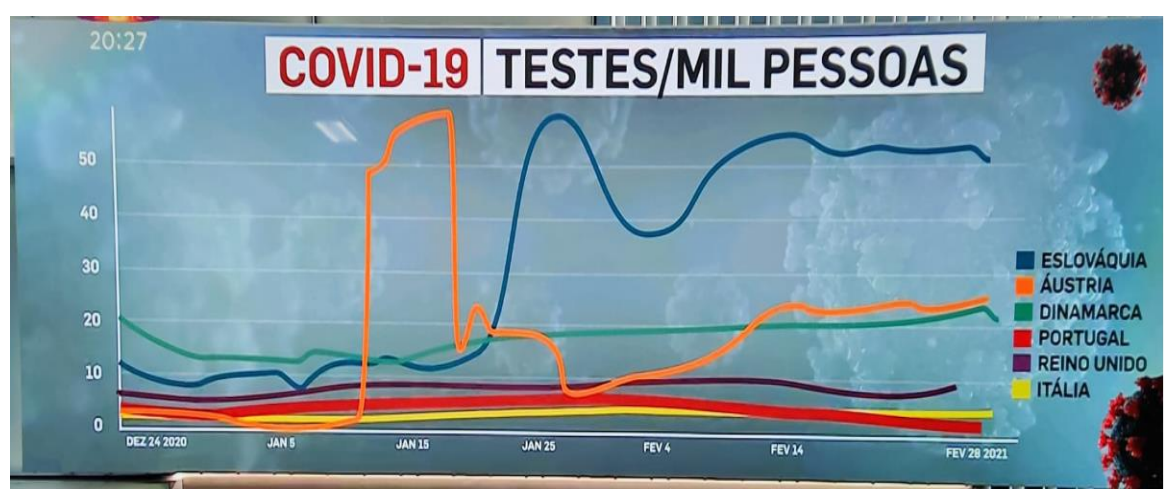

Gráfico 8. Número de testes Covid-19/milhão de habitantes

Em número de vacinados Portugal, pese embora a escassez de vacinas e a problemática que tem envolvido algumas delas, situa-se acima da média europeia (gráfico 9) ${ }^{20}$.

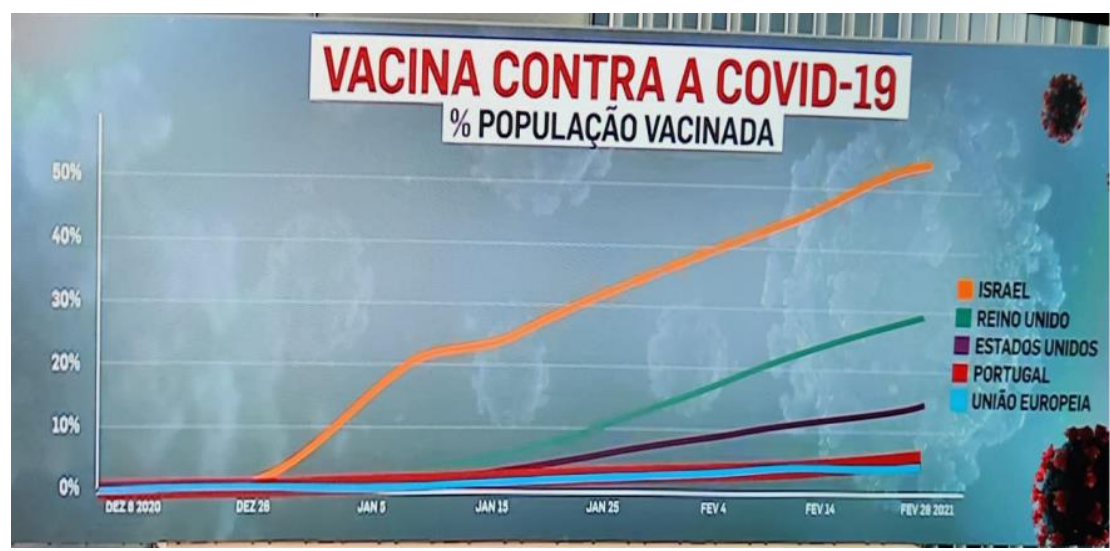

Gráfico 9. Percentagem de população vacinada para a Covid-19.

A vacinação é sem dúvida a esperança no controle da Covid-19, porém as mutações do vírus com o surgimento de novas estirpes ou variantes (Inglesa, Brasileira, Africana e outras) têm colocado apreensão e reservas nas medidas sanitárias e politicas adotadas pelos diversos países. As alternativas às vacinas para pessoas com sistemas imunitários 
comprometidos os anticorpos monoclonais para proteger os milhões de pessoas que não podem ser vacinadas levanta questões em termos do seu custo e viabilidade a longo prazo porém são uma realidade que se apresenta em linha na resposta pandémica.

\section{Reflexões finais}

Globalmente, a Covid-19 atingiu fortemente o setor da saúde e o setor social. Os profissionais de saúde da primeira linha têm-se revelado verdadeiros heróis nesta luta musculada contra o novo vírus SARS-CoV-2. Os avanços científicos, a ampla divulgação para melhor conhecer o vírus e a forma de o controlar, a cooperação internacional, bem como a vacinação e a produção de medicamentos mais eficazes no tratamento têm acompanhado este processo e mantido a esperança no futuro.

\section{Bibliografía}

ALMEIDA, Teresa Caldas de et al, Relatório Final: SM-Covid19 - Saúde Mental em Tempos de Pandemia, Lisboa, Instituto Nacional de Saúde Doutor Ricardo Jorge, 2020.

ANDERSON, RM, HEESTERBEEK, H, KLINKENBERG, D, HOLLINGSWORTH, TD, "How will country-based mitigation measures influence the course of the COVID19 epidemic?", Lancet, 2020, 395, 931-34.

BROOKS, Samantha et al, "The psychological impact of quarantine and how to reduce it: Rapid review of the evidence", Lancet, 2020, 395(102227), 912-20. http://doi.org/10.1016/S0140- 6736(20)30460-8

CHEN, Nanshan et al, "Epidemiological and clinical characteristics of 99 cases of 2019 novel coronavirus pneumonia in Wuhan, China: a descriptive study", Lancet, 2020, 395, 507-13.

DEMARTINI, Karine et al. "Care for frontline health care workers in times of COVID19", Rev. Soc. Bras. Med. Trop., 2020, vol.53, e20200358, https://doi.org/10.1590/0037-8682-0358-2020

HOFFMANN, Christian, KAMPS, Bernd Sebastian, COVID Reference, Volume 1, Hamburg, Steinhäuser Verlag, 2020.

HUANG, Chaolin et al, "Clinical features of patients infected with 2019 novel coronavirus in Wuhan, China", Lancet, 2020, 395, 497-506.

KSIAZEK, TG et al, "A novel coronavirus associated with severe acute respiratory syndrome". N Engl J Med. 2003, 348, 1953-1966.

LAI, Jiambo et al, "Factors Associated With Mental Health Outcomes Among Health Care Workers Exposed to Coronavirus Disease 2019”, JAMA, 2020, 3(3), e203976, https://doi.org/10.1001/jamanetworkopen.2020.3976. 
NGUYEN, LONG et al. "Risk of COVID-19 among front-line health-care workers and the general community: a prospective cohort study", Lancet Public Health, 2020, 5, e475-83.

RODRÍGUEZ-REY, Rocío, GARRIDO-HERNANSAIZ, Helena and BUENOGUERRA, Nereida, "Working in the Times of COVID-19. Psychological Impact of the Pandemic in Frontline Workers in Spain". International Journal of Environmental Research and Public Health, 2020, vol. 17, 21 8149, doi:10.3390/ijerph17218149

ROGERS, Jonathan, CHESNEY, Edward and OLIVER, Dominic, "Psychiatric and neuropsychiatric presentations associated with severe coronavirus infections: a systematic review and meta-analysis with comparison to the COVID-19 pandemic", Lancet Psychiatry. 2020, 7(7), 611-627, ht tps://doi.org/10.1016/S22150366(20)30203- 0

\section{Anexo}

AMNISTIA INTERNACIONAL, PUBLIC SERVICES INTERNATIONAL E UNI GLOBAL UNION. Health Workers Deaths Due to COVID-19, https://www.amnistia.pt/covid-19-17-mil-profissionais-de-saude-morreram-em-2021/

GENDER DIVERSITY INDEX. European Women on Boards Gender Diversity Index, 2020.

Organização Internacional do Trabalho (OIT), Trabalho digno em Portugal 2008-2018: da crise à recuperação, Genebra, BIT, 2018.

REPÚBLICA PORTUGUESA, ADMINISTRAÇÃO INTERNA, Relatório sobre a Aplicação da Declaração do Estado de Emergência 16 a 30, de janeiro de 2021, Estratégia de aplicação das medidas de combate à pandemia da doença COVID-19, https://www.parlamento.pt/Documents/2021/fevereiro/Relatorio-Estado-Emergencia16-30jan.pdf

REPÚBLICA PORTUGUESA, SERVIÇO NACIONAL DE SAÚDE. Relatório Social Do Ministério Da Saúde E Do Serviço Nacional De Saúde 2018, https://www.sns.gov.pt/wp-content/uploads/2019/09/Relat\%C3\%B3rio-SocialMS_SNS-2018-002.pdf

SIC, Jornal da noite, 2 de março de 2021.

World Health Organization, Health Emergency Dashboard https://covid19.who.int/region/euro/country/pt

World Health Organization, Novel Coronavirus - Thailand, 2020. 